\title{
Biological information processing: the use of information for the support of function
}

\author{
Roberto R. Kampfner \\ School of Management and Computer and Information Science. University of Michigan-Dearborn, Dearborn, MI 48128 \\ (U.S.A.)
}

(Received September 22nd, 1988)

(Revision received October 21st. 1988)

\begin{abstract}
In biological systems, the processing and use of information has evolved out of the need for survival in the face of an uncertain environment. As a consequence, the information-function relationship in these systems is shaped by their adaptability characteristics. In contrast, the information-function relationship in man-designed, goal-oriented organizational systems depends on the ability of the information processing system to support the achievement of the organization's goals. In this paper we use results from adaptability theory in the analysis of control-related aspects of the information-function relationship in man-designed organizational systems. In particular, we use a conceptual model of organizational control to characterize features of functional and control structures and their effect on the adaptability of these systems. The concept of implicit control and a design principle for adaptability-enhancing information systems are derived for this analysis.
\end{abstract}

Keywords: Adaptability: Organizational systems; Information systems; Biological information processing.

\section{Introduction}

Processing and use of information in biological systems can be said to have evolved out of the need for survival in the face of an uncertain environment. Accordingly, biological information processing can be said to support function to the extent to which these systems are able to adapt to their natural environments. In our view, any aspect of function in biological systems is related in a natural way to the overall system's behavior. In this sense, although individual functions can be identified in many specific contexts, their contribution to overall system's adaptability and function is always an essential characteristic. The degree of adaptation of an organism, or group, at any level of biological organization, is thus contingent upon the characteristics of its interaction with many other organisms or groups at different levels. Moreover, it is especially contingent upon how it affects their degree of adaptation. Mechanisms and strategies for adaptability and their impact on biological information processing are, therefore, most relevant for the characterization of this interaction.

In goal-oriented organizational systems, on the other hand, information processing is normally planned and designed for the achievement of specific goals. Because of the nature of this design effort, however, the organizational structures so designed and, in particular, their supporting information systems, are not necessarily consistent with their declared goals, nor with their adaptability requirements. In this paper we use results from systems theory (Laszlo, 1972) and adaptability theory (Conrad, 1983) to derive an information systems design principle for goal-oriented, organizational systems. More precisely, we define a notion of implicit control in a system, as opposed to that explicitly exerted by a dedicated control subsystem. Our design principle concerns the 
adequate use of implicit control so that an effective use of information processing is achieved without loss of adaptability.

The view of organizations as hierarchical arrangements of functional subsystems, each responsible for a specific subgoal, but acting in concert for the achievement of the overall goals of the organization is vital to this endeavor. We use the organizational control systems model (OCSM), a hierarchical model of organizational control (Kampfner, 1987) that formalizes the representation of organizational control structures based on the hierarchical systemic view, as a tool for the analysis of specific organizational structures.

This paper is organized as follows. Biological information processing is discussed in $\$ 2$ from the viewpoints of natural systems and the adaptability theory framework. Section 3 discusses the role of the OCSM in the analysis of adaptability properties of organizational systems from the point of view of information processing. Our design principle for adaptability-enhancing organizational information systems is discussed in $\$ 4$

\section{Biological information processing}

\subsection{The Natural Systems Framework}

Current knowledge suggests that natural systems have evolved into what we interpret as a systemic hierarchy, including living and non-living systems. There are many ways in which such a hierarchy can be defined. Boulding (1956), for example, considers features of systems ranging from static structures, at the lowest level, through simple dynamic systems, control mechanisms, and open systems or self-maintaining structures, up to man, social organizations and other higher-level suprasystems. Laszlo (1972) uses a hypothetical identification of the principal levels and interrelations of micro- and macro- hierarchies, and proposes four joint, in dependent variables, as underlying the behavior of systems at all levels in the microhierarchy, which includes levels ranging from the atom, to the ecosystem, to the world system. These joint, independent variables are: (i) wholeness and order: (ii) adaptive selfstabilization; (iii) adaptive self-organization; and (iv) systemic hierarchy. We find these joint, independent variables of natural systems most relevant to our study of the structure and essential dynamics of organizational systems and their adaptability properties

Properties corresponding to these variables can, indeed, be identified at all levels of biological organization. At the macro-molecular level, for example, all of the components of a protein enzyme, such as its constituent atoms and the bonds between them, determine its three-dimensional structure, hence its function (Conrad, 1985b). Protein function itself acquires a definite meaning only when the context in which the substrate on which it acts, and the milieu in which such substrate finds itself, are specified. Selfstabilization and self-organization properties can. of course, be easily discerned in connection with protein function and evolution. Lastly, from the viewpoint of systemic hierarchy, the idea of protein enzymes as subsystems, or components, of higher level systems, and themselves composed of smaller subsystems is of course a familiar one. Properties corresponding to the four joint, independent variables of natural systems can also be identified at higher levels of biological organization. including the cell, organism, population. community, and ecosystem level

One of the main characteristics of biological systems is that they adapt to their environments in order to maintain a pattern of organized activity. Moreover, their adaptability implies a potential ability to change their pattern of organization, that is. to evolve. In the next section we identify important aspects of the information-function relationship from the point of view of adaptability theory. 


\subsection{The Adaptability Theory Framework}

According to Ashby's principle of requisite variety (Ashby, 1956), adaptable systems must have a sufficiently large repertoire of behaviors in order to be able to cope with an uncertain environment. Conrad's adaptability theory (Conrad, 1983) analyzes the strategies followed by biological systems in order to maintain, and evolve, well-defined patterns of activity. Adaptability theory helps us understand the nature of the mechanisms used by biological systems as they adapt to their environments and helps us identify their basic constraints and strategies. These mechanisms, constraints, and strategies constitute an important, underlying basis of the information-function relationship in biological systems. We will consider some aspects of this relationship from the point of view of adaptability theory in this section.

Conrad's formalism defines adaptability in terms of the potential uncertainty of the transition scheme of a biological system, and its relationship to the uncertainty of the transition scheme of its environment. Entropies and conditional entropies, defined on the basis of these transition schemes, are related according to the following fundamental inequality (Conrad, 1983):

$$
U(S)-U(S \mid E)+U(E \mid S) \geqslant U(E)
$$

Here, $U(S)$ represents the behavioral uncertainty of system $S, U(E)$ represents the behavioral uncertainty of the most uncertain environment that does not cause a catastrophic change in $S, U(S \mid E)$ represents the degree of correlation of the behavior of $S$ with respect to the behavior of its environment, and $U(E \mid S)$ represents the degree of correlation of the behavior of the environment with respect to $S$ 's behavior. The term $(U(S)-U(S \mid E))$ is interpreted as a measure of the extent to which $S$ anticipates the behavior of the environment, and $U(E \mid S)$ as a measure of the indifference of $S$ with respect to the behavior of the environment.
A concept relevant to adaptability theory, but which is also extremely important from the point of view of the information-function relationship is the effectiveness of the use of information processing that a system possesses. Our interpretation of this concept is that a system makes effective use of information processing to the extent to which it is capable of making decisions leading to adequate responses to its environment. This effectiveness is thus strongly related to the control and decision-making capabilities of systems. It is also strongly related to the anticipation component of adaptability. Essentially, what adaptability theory says, in this respect, is that the effective use of information processing in a system reduces the number of allowable trajectories required for its adaptability.

Two important aspects of a system's structure and behavior that are especially relevant from the viewpoint of adaptability are the degree of independence between its subsystems and the predictivity of its transition scheme. A related important result is that the predictivity of the transition scheme of a subsystem which is independent from other subsystems actually depends on the adaptability of such subsystems.

A third result concerns the hierarchical, or compartmental organization of a system. In this respect, the need for compensation of losses in adaptability calls for the possibility of allocating in a selective manner the required adaptability to other parts of the system. Clearly, a hierarchical or compartmental organization provides a convenient vehicle for the effective distribution of additional adaptability

The results from adaptability theory mentioned above can be summarized, from the point of view of the impact of information processing on the adaptability of a system. as follows: from an adaptability viewpoint, the goal of an information processing system is to increase the effectiveness of the use of information processing without reducing overall system's adaptability. This statement 
implies the existence of complex trade-off's that must be adequately balanced for a system to function effectively. In the case of biological systems such trade-offs have been taken care of by natural selection through the course of biological evolution. From the standpoint of man-designed, goal-oriented organizational systems, the implication of the results mentioned above is that, in order for an organizational system to be adaptable. both organizational design and the design of the supporting information system must be consistent with that particular system's goals. These goals and designs must, of course, also be consistent with the organization's potential for adaptability

\section{A hierarchical model of organizational control}

The organizational control systems model, or OCSM (Kampfner, 1987), is based on the idea of organizations as goal-oriented, adaptive systems having a hierarchical control structure. These systems are assumed to possess the four joint, independent variables of natural systems mentioned above. The importance of closely related hierarchical control loops in organizations, and their impact on their information processing needs, has been discussed in the context of organizational design by Gerloff (1985). Here we use the OCSM as a conceptual tool for the application of the results of adaptability theory mentioned above to the analysis of the adaptability properties of organizational structures, their information needs, and information processing requirements. This analysis will help us define a basic principle for the design of the supporting information processing systems.

Formally, a goal-oriented organizational system is a structure

$O:=\langle S, R\rangle$

where

$S=\left\{s_{t, j}\right\}$ is a set of functional subsystems

$R=\left\{\rho_{s}, \rho_{c}, \rho_{0}\right\}$

is a set of relations on $S$. Here, $\rho_{8} \subseteq S \times S$ is the subsystem relation. More precisely, $s_{i, j} \rho_{s} s_{i+1, k}$ means that $s_{i, j} \in S$ is a subsystem of $s_{i+1, k}$ (see Fig. 1).

We will also distinguish $S_{n} \subseteq S$, the set of control subsystems in $S$. An important feature of OCSM is that, in this model, each functional subsystem $s_{i, j} \in S$, has a distinguished control subsystem $c s_{i} \quad{ }_{1, m_{0}}$ and one or more operational subsystems $s_{i}, \mathrm{~m}$.

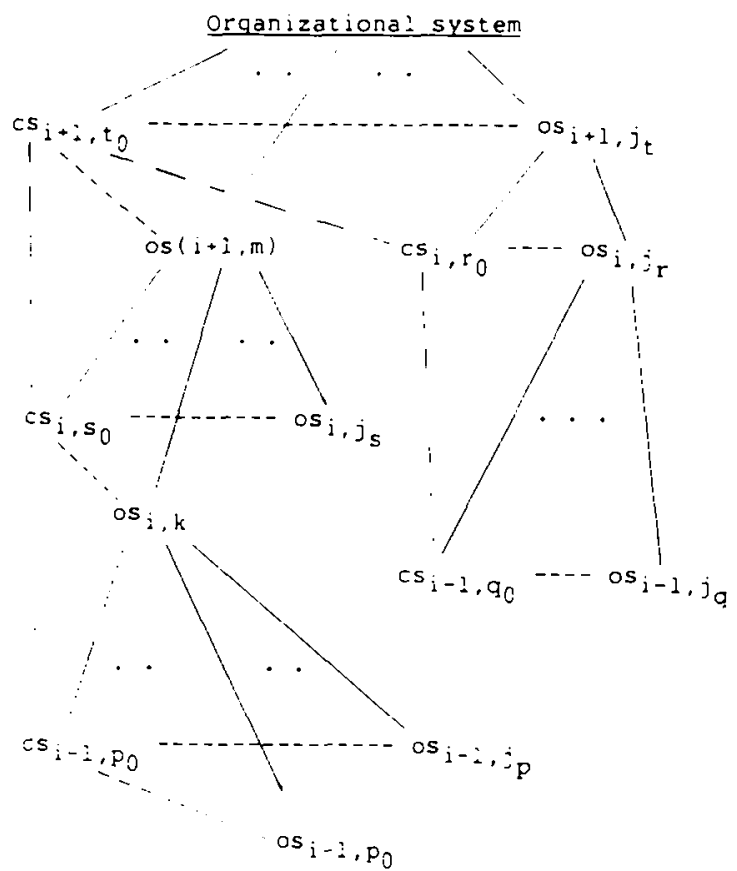

Fig. 1. Schematic view of the relationship between functional and control hierarchies in a goal-oriented, organizational system. Formally, this relationship is expressed as $\rho_{0}=\rho_{\mathrm{c}} \cdot \rho_{\mathrm{g}} \mid S_{0}$. — joins elements of a pair in relation $\rho_{8} ;-\cdot-\cdot-$ joins elements of a pair in relation $\rho_{0},----$ joins elements of a pair in relation $\rho_{c}$. A control subsystem, $c s_{t, m}$ say, represents the internal control of its (operational) parent subsystem. $o s_{i \cdot 1, \mu}$, in the case of $c s_{i, m}$. Its responsibility is to coordinate the performance of its parent's operational subsystems, like $O S_{i, 3 r}$. From the point of view of adaptability theory, a control subsystem represents the formal anticipation component of its parent system 
The relationship between control and operational subsystems is formalized in terms of relation $\rho_{c} \subseteq S \times S_{0}$, where $s_{i} 1, m \rho_{c} c s_{i, 1, m_{0}}$ means that $s_{i-1, m}$ "is controlled" by $c s_{i \cdot 1, m_{0}}$. Figure 1 also shows, schematically, relation $\rho_{c}$.

Another important relationship is that between control subsystems at contiguous levels in the hierarchy. This relationship is formalized by relation $\rho_{0} \subseteq S_{0} \times S_{0}$. Here, $c s_{i}{ }_{1, m_{0}} \rho_{0} c s_{i, j_{0}}$ means that $c s_{i-1, m_{0}}$ "reports to" $c s_{i, j_{1}}$. In this case, $c s_{i}{ }_{1, m_{0}}$ is such that both $c s_{i} \quad 1, m_{0} \rho_{s} s_{i, j}$ and $s_{i, j} \rho_{c} c s_{i, j_{o}}$ hold.

The relationship between these relations can also be expressed formally as

$\rho_{0}=\left.\rho_{c} \cdot \rho_{\mathrm{s}}\right|_{\mathrm{s},}$

where $\left.\rho_{s}\right|_{s,}$ is the restriction of $\rho_{s}$ to the control subsystems in its codomain. Figure 1 also illustrates this relationship.

Essentially, the OCSM portrays goaloriented organizational structures with a hierarchical control system. Organizational structures mapped into the OCSM are adaptable, and do incorporate the four joint, independent variables of natural systems described above. In what follows, we use the OCSM as a reference structure for the study of adaptability properties of goal-oriented organizational systems from the standpoint of the structure of their control systems and their information processing characteristics.

A basic parameter of a control subsystem of an organization modelled with the OCSM is its scope. This parameter defines the degree of centralization/decentralization of an organization's control system. There are various kinds of information flows implied by the OCSM. First, we have vertical information flows that interconnect control systems at contiguous levels in the hierarchy. Information flowing down consists of goals and parameters assigned by each control subsystem to each of its controllees. Information flowing up consists of the necessary feedback returned by each control subsystem to its corresponding controller about the actual performance of its parent subsystem. These flows are actually processed by the control systems as part of the control function of each subsystem being controlled. This is formalized by relations $\rho_{s}, \rho_{r}$ and $\rho_{0}$ described above.

We define the adaptability of organizational systems in terms of the structure of their control systems as follows.

The anticipation component of the adaptability of $s_{i . m}$, an organization's functional subsystem, can be expressed as

$$
A_{\mathrm{s}}=U\left(\bar{s}_{i, m}\right)-U\left(\bar{s}_{i, m} \mid \bar{e}_{i, m}\right)
$$

where $\bar{s}_{i, m}$ is the transition scheme of operational subsystem $s_{i, m}$ and $\bar{e}_{i, m}$ denotes the transition scheme of $e_{i, m}$, the environment of $s_{i, m}$.

We can also define the relative anticipation of control subsystem $c_{i} \quad{ }_{1, j_{0}}$ as

$$
A_{\mathrm{c}}=U\left(\bar{c}_{i, 1, j_{0}}\right)-U\left(\bar{c}_{i-1, j_{0}} \mid \bar{e}_{i, m}\right)
$$

with $c_{i} \quad{ }_{1, j_{0}}$ a subsystem (the internal control) of $s_{i, m}$, i.e. $c_{i-1, j_{0}} \rho_{8} s_{i, m}$.

Briefly, the relative anticipation measures the degree to which the behavior of control subsystem $c_{i-1, j_{0}}$ is correlated with that of $e_{i, m}$, its parent system's environment.

Let $c_{k} \in S_{0}$ denote a control subsystem in the OCSM hierarchy. The scope of a control subsystem $c_{k}$ is then defined as

$u_{k}=A_{c} / A_{s}$

Let $k \in I$ be an index running over all the control subsystems of an organizational system, i.e. $k \in\{1, \ldots, m\}$, where $\left.m=S_{0}\right\}$. The degree of centralization of an organizational control system is thus expressed as

$\Psi_{c}=\sum_{k} u_{k} \cdot i_{k} / m$

where $i_{k}$ is the level of control subsystem $c_{k}$ in the control hierarchy.

Hierarchical control levels are defined as 
for the OCSM (cf. $\$ 3$, above). The highest control level corresponds to top management in a business organization. for example. A basic assumption of the OCSM is that organizational goals, as seen by top management, for instance, can be subdivided into a set of subgoals, each of which corresponds to a functional subsystem with its own internal control. Each of these functional subsystems can, in turn, be subdivided, etc.

$u_{k}$ measures the degree to which control subsystem $c_{k}$ monitors the responses of its parent system to its environment. High $u_{k}$ means that a correspondingly large percentage of the actions that system $s_{\iota, m}$ undertakes in order to respond to its environment are in fact directed and monitored by its control subsystem. $\Psi_{\text {, }}$, the degree of centralization of an organizational control system, is in fact a weighted average of anticipation measures $u_{k}$ for each subsystem of the organization. Here, higher level control subsystems are given more weight than lower level ones. This means that the greater the value of $\psi_{c}$, the greater the degree of centralization of the overall organization's control system. $\Psi$, thus measures the degree to which the anticipation component of adaptability or, equivalently, the information processing and decision-making capability of the system, is allocated to higher levels in the control hierarchy.

\section{A design principle for adaptability- enhancing organizational information systems}

By an organizational information system we mean the formal means of information processing operating in a man-designed organizational system such as a business organization. The idea here is to apply the results from adaptability theory mentioned above to man-designed, organizational systems. As mentioned earlier, an adaptabilityenhancing information system should increase the effectiveness of information processing at the lowest possible cost. It was also noted that this implies that adequate trade-offs must be achieved between the cost of information processing, the cost of maintaining a larger number of trajectories for the system, and the cost of adaptability. In goal-oriented organizational systems, the goals themselves constrain the ways in which adaptability can be increased. These constraints apply, for instance, to the indifference component of the system, especially when the goals imply some required degree of interaction between the system and its environment. The indifference component of adaptability (Conrad, 1983) represents the degree to which an adaptable system can be indifferent to the behavior of the environment. Formally, the indifference component is expressed as $U\left(\bar{e}_{i, j} \mid \bar{s}_{i, j}\right)$, for subsystem $s_{i, j}$, and its environment $e_{i, j}$

The implicit control concept. Let us first consider the problem of increasing the effectiveness of information processing. It is important to notice, in this respect, that the control subsystem (or internal control) of a system carries, by definition. the responsibility of making information processing effective. To this effect, the basic goal is to increase the anticipation component of the system's adaptability. However, since we are concerned with a hierarchical control structure. this responsibility may be distributed in different ways throughout the various control subsystems. In particular, the lowest level in the hierarchy is composed of operational subsystems that do not have an internal control. These subsystems have their control mechanisms embedded in their dynamics. This is reminiscent of biochemical and physiological mechanisms of regulation and control in biological systems. In man-designed, organizational systems one can think of this kind of "implicit control" whenever such control mechanisms are embedded in the dynamics of the process being controlled, whether it is mediated by symbolic information processing or not.

We will refer as implicit control to the col- 
lection of control mechanisms existing in a system but which, for a particular subsystem, are not explicitly recognized as part of its (internal) control. Clearly, the implicit control concept is a relative one, since what is a control subsystem at some level, may be considered, from the point of view of a higher level, as implicit control. However, at any given level, the implicit control of a system resides in its operational subsystems, as opposed to its dedicated (internal) control subsystem. Thus, for example, a financial manager in a business organization can be thought of as playing a key role within the control subsystem of an organization's financial system (itself a subsystem of the organization). In that capacity he can establish policies and set specific goals to an operational subsystem responsible, say, for billing customers and collecting payments in as effective a manner as possible. The control mechanisms used within the billings and collections subsystem may not be visible to the financial manager and, in that case, can be regarded as part of the implicit control of the financial subsystem. In the case of biological systems, taking man as an example, one can think of the conscious brain as the dedicated, explicit control system. Implicit control, on the other hand, would involve mechanisms such as those controlling body temperature, metabolism, etc. Formally, an increase in implicit control corresponds to a decrease in $\Psi_{c}$, the degree of centralization of the organizational control system, but subject to trade-offs, as explained below.

We can now state a basic design principle for man-designed, organizational information systems: an information system that makes adequate use of the implicit control that is possible in an organizational system allows for an effective use of information processing at a relatively low cost and without loss of adaptability. By an adequate use of implicit control we mean that the trade-offs obtained between the cost of the effective use of information processing, the cost of maintaining a large number of allowable number of trajectories required for the system, and the cost of adaptability, are consistent with this principle, that is, the total cost involved is relatively low, and there is no loss of adaptability. Clearly, this principle also applies to the information processing aspects of organizational design.

For this design principle to be really useful we need to indicate in more detail specific strategies for the reduction of each of these types of costs.

Reducing the cost of information processing. To reduce the cost of increasing the effectiveness of the use of information processing, the cost of information processing itself must be reduced. This in turn requires that the complexity of the control function in the system be reduced. This can be achieved by increasing, as much as possible, the degree of implicit control of operational subsystems at all levels, while increasing the anticipation component of the whole system. However, this may cause the existence of very independent subsystems, with highly predictive transition schemes. This brings with it a loss of adaptability. The cost of compensating for lost adaptability, therefore, imposes a limit on the degree of implicit control that can be tolerated in the various operational subsystems of the system.

Reducing the cost of maintaining a large number of allowable trajectories. According to adaptability theory, increasing the effectiveness of the use of information processing reduces the number of allowable trajectories required by the system. This brings a reduction in the cost of maintaining a large number of such trajectories. However, increasing the effectiveness of the use of information processing carries a cost and may cause a loss of adaptability if the complexity of the system increases.

Reducing the cost of adaptability. This cost basically occurs as a loss of efficiency of adaptability that is caused by an increase in the interdependence between system components. Although the argument against complexity has been put forth in the context 
of biological evolution, it seems reasonable to assume that it also applies to the adaptability of man-designed, goal-oriented, organizational systems. As mentioned above, an increase in the effectiveness of the use of information processing may increase this cost as a result of the compensation of lost adaptability if, again, this compensation is not properly guided. The impact of the increase of effective information processing on adaptability is explored by Conrad in relation to the effect of programmability on evolutionary plasticity (Conrad, 1985a,b).

We finish our discussion on the principle of design for organizational information systems by saying that the trade-offs mentioned can be assessed accurately enough, only in terms of evaluations of the underlying costs in specific systems.

\section{Summary and conclusions}

Our main result is a design principle for adaptability-enhancing information systems in man-designed organizations, derived from results from adaptability theory, originally formulated in the context of biological evolution. that we then applied to the study of adaptability in man-designed organizations with the help of a hierarchical model of organizational control. This principle asserts that the effectiveness of the use of information processing in these systems can be increased, at a relatively low cost and without loss of adaptability, provided that an adequate use of implicit control is made. To derive this principle we developed the notion of implicit control, which represents control mechanisms embedded into the dynamics of the controlled system itself in a manner reminiscent of biochemical and physiological control in biological systems. In the context of man-designed, goal-oriented organizational systems, implicit control refers to control mechanisms that are not visible at the level of explicit, formal control. We also developed a measure of the degree of centralization of control in an organization, on the basis of the distribution of the anticipation component of adaptability throughout the organization's control hierarchy, and related it to trade-offs between the costs associated with the increase of effectiveness of information processing

Although basic criteria for the adequate use of implicit control are discussed, it is also acknowledged that the actual parameters to be considered in specific cases depend on characteristics specific to a given organizational system and its environment.

Another important conclusion from the study of the adaptability characteristics of man-designed, goal-oriented organizational systems is that information system support of specific organizational functions, or subsystems, cannot be provided effectively without considering its contribution to the adaptability of the organization as a whole.

\section{Acknowledgements}

This research was supported, in part, by NSF Grant IRI-87-02600.

\section{References}

Ashby, W.R, 1956, An introduction to cybernetics (Wiley, New York)

Boulding, K.. 1956, General systems theory: the skeleton of science. Manage. Sci.. April 1956, pp. 197-208

Conrad, M. 1983. Adaptability (Plenum Press. New York).

( onrad. M.. 1985a. Adaptability theory as a guide for interfacing computers and human society, in: 1985 AAAS Symposium on Recent Integrations of Research on Biological and Social Living Systems. J. Miller (ed.), in press

('onrad. M., 1985b. On design principles for a molecular computer. Commun. ACM 28. 464-480.

Gerloff, E.. 1985. Organizational theory and design (McGraw-Hill, New York)

Kampfner R, 1987, A hierarchical model of organizational control for the analysis of information systems requirements. Inf. Syst. 12. 243-254.

Laszlo, E.. 1972, Introduction to systems philosophy (Harper and Row. New York) 